\title{
ANÁLISIS DEL RENDIMIENTO EN EL DOCTORADO EN FUNCIÓN DEL SEXO
}

\author{
(SEX-BASED PERFORMANCE ASSESSMENT IN DOCTORAL DEGREES)
}

M. Paz Bermúdez, Alejandro Guillén-Riquelme, Almudena Gómez-García, Raúl Quevedo-Blasco, Juan Carlos Sierra y Gualberto Buela-Casal

Universidad de Granada

\section{RESUMEN}

Introducción: Las mujeres no están representadas de manera igualitaria respecto a los hombres a lo largo de las categorías profesionales del sistema científico. Varios estudios demuestran que el primer escalón donde se verifica una disminución del número de mujeres es el doctorado. El objetivo de este estudio era evaluar el rendimiento global y por área de conocimiento en el doctorado en función del sexo. Método: Como medida de rendimiento, se comparó el número de tesis doctorales registradas en la base de datos de Tesis Españolas Ordenadas TESEO del Ministerio de Educación del Gobierno de España entre 2002 y 2009 por sexo en una muestra 3.463 doctorandos (1.724 mujeres, 1.739 hombres). Resultados: El análisis del rendimiento diferencial por sexo no mostró diferencias estadísticamente significativas a nivel global ni por áreas de conocimiento. Conclusiones: El presente estudio confirma que, cuando los doctorandos son beneficiarios de una beca predoctoral y en condiciones igualitarias, no existen diferencias de rendimiento en programas de doctorado en función del sexo.

\section{ABSTRACT}

Introduction: Unlike men, women are not equally represented in the different professional categories of the scientific system. According to several studies, the first noticeable decrease in the number of women takes place in 
doctoral degrees. The aim of this study was to evaluate sex differences in performance in doctoral degrees both globally and by areas of knowledge. Method: As a measure of performance, the number of doctoral theses registered in the Ordered Spanish Theses (TESEO) data base between 2002 and 2009 was compared across sex in a sample of $3.463 \mathrm{PhD}$ students (1.724 women, 1.739 men). Results: The differential analysis did not show any statistically significant difference either at the global level or any specific area of knowledge. Conclusion: This study confirms that, when PhD students are given a pre-doctoral scholarship, there is not any sex difference in performance in doctoral degrees.

\section{INTRODUCCIÓN}

La condición de la mujer en el sistema de educación y ciencia es un tópico que ha estimulado un amplio debate internacional. Entre los primeros estudios que denunciaban la escasez de mujeres en la investigación científica se encuentra un informe de Handschin (1911), publicado en la revista Science, donde se constataba la escasez de profesoras en las instituciones educativas en rangos profesionales elevados (Handschin, 1911). Cattel, profesor de la Universidad de Columbia, estadístico y exponente de las teorías sociológicas orientadas biológicamente, explicaba que la escasa presencia de la mujer en la ciencia era debida a su supuesta inferioridad intelectual (tal como se cita en Pérez, et al., 2003).

Desde entonces la condición social de la mujer ha mejorado mucho. Algunos pasos importantes hacia la igualdad entre sexos han sido la Carta de las Naciones Unidas (Asamblea General de las Naciones Unidas, 1945), la Declaración Universal de los Derechos Humanos (AGONU, 1948) y la Convención sobre la eliminación de todas las forma de discriminación contra las mujeres (Consejo Económico Social y de las ONU, 1979). El documento más importante y significativo en Europa sobre este tema es el Informe ETAN, que denuncia la injusticia, además de la amenaza para la excelencia, de una representación tan baja de la mujer en el sistema científico europeo (Osborn, 2001).

En España, la igualdad entre hombre y mujer está establecida por el artículo 14 de la Constitución. En 2002 la Junta del Gobierno del Consejo Superior de Investigación Científica aprobó la constitución de una comisión para "el estudio de los problemas que dificultan la carrera científica de las mujeres en el CSIC" (CSIC, 2002) y en 2003 fue creada la Asociación de Mujeres Investigadoras y Tecnólogas. De manera similar la Fundación Española para la Ciencia y la Tecnología tiene abierta desde sus inicios una línea de trabajo sobre el papel de las mujeres en la actividad científica con finalida- 
des similares. Con la Orden del 7 marzo 2005 (Ministerio de la Presidencia, 2005) se adoptaron medidas para favorecer la igualdad entre mujeres y hombres y se creó la Unidad de Mujeres y Ciencia, dependiente del Ministerio de Educación y Ciencia, para vigilar la situación de la mujer en la investigación. Finalmente, la Jefatura del Estado (2007), establece mediante Ley Orgánica, lo siguiente:

"Resulta necesaria, en efecto, una acción normativa dirigida a combatir todas las manifestaciones aún subsistentes de discriminación, directa o indirecta, por razón de sexo y a promover la igualdad real entre mujeres y hombres, con remoción de los obstáculos y estereotipos sociales que impiden alcanzarla". (p. 12611)

El número de alumnas en la enseñanza primaria, media o secundaria y en la universidad, así como de profesoras, ha crecido de manera constante en las últimas décadas si contamos que en los años 40 en España las universitarias eran el 13\% del total (Pérez, González, Miranda, Ortega y Sanz, 2006) y que en el año académico 1972-73 constituían el 31\%, donde el $40 \%$ estudiaba humanidades y solamente el $2 \%$ ciencias técnicas (Pérez, 1996). Hasta los años 80 las mujeres no llegaron al 50\% de los estudiantes que terminan la enseñanza universitaria (De Pablo, 2004), constituyendo en los años 1990-1991 el 57,8\% y en 2003-2004 el 59,10\% de todos los licenciados (Pérez et al., 2006). En el Informe PISA de la Organización para la Cooperación y el Desarrollo Económico (2003) se afirma que las mujeres están por delante de los hombres en todos los niveles del sistema educativo excepto en las carreras técnicas, terminan con éxito sus estudios universitarios y su abandono es escaso, pero en los datos relativos a su participación en los niveles más prestigiosos del sistema científico no se mantienen estas proporciones (FECYT, 2005). Por ejemplo, en el año académico 2007-2008 las mujeres matriculadas en la universidad pública constituían el 60\% del total, el 65\% de los licenciados y el 52\% de los matriculados en los estudios de doctorado (Instituto Nacional de Estadística, 2009).

Pese a que el número de estudiantes mujeres y licenciadas sea mayor que el número de hombres, de estas mismas características, la proporción entre hombres y mujeres se desequilibra a lo largo de la carrera investigadora (CSIC, 2009; MEC, 2007). La figura 1 representa esta situación, donde el número de mujeres disminuye al aumentar el prestigio del puesto de trabajo del sistema científico, trazando un modelo de tijera. 


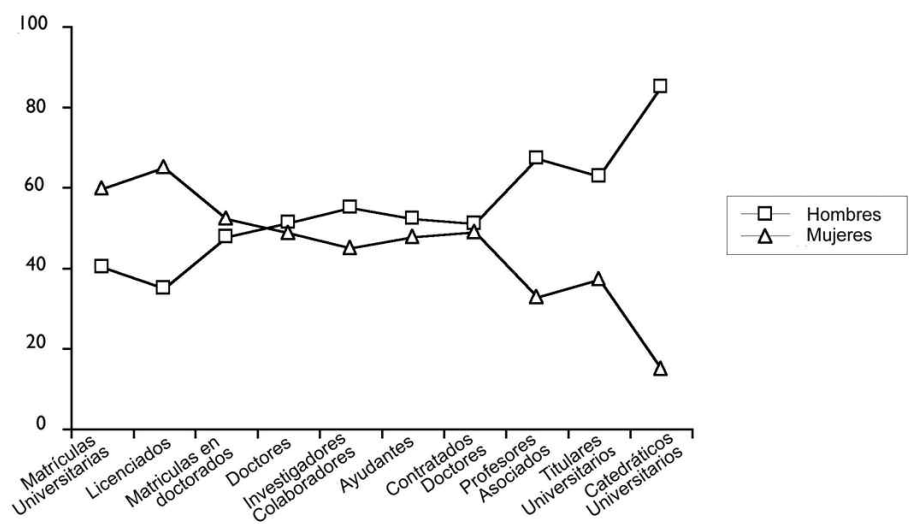

Figura 1. Distribución de hombres y mujeres en los distintos niveles de la carrera académica universitaria

En varios trabajos se indica que el primer nivel académico donde se registra una disminución de capital humano femenino es el doctorado (De Pablo, 2004; Pérez et al., 2006). A pesar de que las mujeres matriculadas sean más numerosas respecto a los hombres (el 52\% del total), solamente llegan a terminar el 49\% de las tesis doctorales en el año académico 2007-2008 (INE, 2009).

Existen múltiples estudios que han analizado la evolución de las Escuelas Doctorales (BuelaCasal y Castro 2008a; Castro et al., 2010) y muchos autores han evidenciado la importancia del doctorado, el grado académico más importante del sistema educativo y puerta de acceso a la carrera investigadora para la evaluación de la productividad científica tanto de las universidades (Agudelo et al., 2003; Bermúdez, Castro, Sierra y Buela-Casal, 2009; Buela-Casal 2005; Buela-Casal y Castro, 2008b; Castro y Buela-Casal, 2008; Moyano, Delgado-Domínguez y Buela-Casal, 2006), como del profesorado (Buela-Casal, 2010; Buela-Casal y Sierra, 2007; Olivas-Ávila y MusiLechuga, 2010; Sierra, Buela-Casal, Bermúdez y Santos-Iglesias, 2009b). En este sentido también se han realizado trabajos sobre la productividad de los programas de doctorado (Musi-Lechuga, Olivas-Ávila y Buela-Casal, 2009; Quevedo-Blasco, Del Río, Bermúdez y Buela-Casal, 2010) y en concreto sobre el rendimiento en el doctorado de los becarios de Formación del Profesorado Universitario (FPU) y Formación de Personal Investigador (FPI) (Guillen-Riquelme, Guglielmi, Ramiro, Castro y Buela-Casal, 2010), además de analizarse aspectos relacionados con la contratación de investigadores (Bretz, 2010). En otros trabajos se ha mostrado que los estudios de doctorado tienen una baja tasa de éxito (Buela-Casal, 2005; González-Ra- 
mos, et al., 2006). Según los datos aportados por el INE, en el año académico 2007-2008 de cada 100 alumnos matriculados en el doctorado solamente 11 obtuvieron el título de doctor en aquel mismo año (INE, 2009).

A la luz de lo expuesto hasta aquí y de la importancia del doctorado en la sociedad del conocimiento este estudio se propone analizar el rendimiento global y por áreas de conocimiento de los doctorandos en función del sexo.

\section{MÉTODO}

\subsection{Participantes}

La muestra estaba compuesta por 3.463 doctorandos, de los que 1.739 eran hombres y 1.724 mujeres. Se incluyeron solamente estudiantes titulares de una beca predoctoral de la Agencia General de Estado, con el fin de obtener una muestra homogénea en cuanto a condiciones económicas y de trabajo para la realización de la tesis. Por tanto, 1.517 participantes pertenecían al programa de Formación de Personal Investigador (FPI), de los cuales $741(48,8 \%)$ son mujeres y $776(51,2 \%)$ son hombres. Los restantes 1.946 pertenecían al programa de Formación de Profesorado Universitario (FPU), de los cuales $983(50,5 \%)$ son mujeres frente a $963(49,5 \%)$ hombres.

\subsection{Unidades de análisis}

Número de tesis doctorales registradas en la base de datos de Tesis Españolas Ordenadas, TESEO, del MEC del Gobierno de España entre el año 2002 y 2009.

\subsection{Materiales}

Documentación sobre concesiones de becas de doctorado a nivel nacional, extraída de los Boletines Oficiales del Estado correspondientes, donde se publica la lista de concesiones de las becas de las convocatorias de 2002 y 2003. Disponible en la dirección: http://www.boe.es/diario_boe/.

Base de datos TESEO. Disponible en la dirección: https://www.micinn.es/teseo/irGestionarConsulta.do.

Bases de datos del INE sobre matriculados y doctores del curso académico 2007-2008. Disponible en la dirección: http://www.ine.es. 


\subsection{Diseño}

La presente investigación se enmarca dentro de los estudios de tipo expost facto, concretamente un estudio historiográfico bibliométrico según la clasificación de Montero y León (2007). Para la redacción de este texto se han seguido los criterios propuestos por Ramos-Álvarez, Moreno-Fernández, Valdés-Conroy y Catena (2008).

\subsection{Procedimiento}

Se seleccionaron las convocatorias de los programas FPU y FPI de las convocatorias de 2002 y 2003 y se identificaron los nombres completos de los becarios a través de la página web del MEC. Se buscaron los nombres obtenidos en el sistema de gestión de tesis doctorales a nivel nacional TESEO desde 2002 hasta 2009. Se realizaron dos búsquedas, ejecutadas separadamente por dos investigadores, para evaluar el grado de consenso entre ambos. Los resultados obtenidos mostraron que el porcentaje de acuerdo entre las dos búsquedas realizadas fue del 98,6\%. En aquellos casos en los que no existió consenso en la primera búsqueda, se realizó una nueva de forma conjunta, llegando finalmente a un consenso en el $100 \%$ de los casos. Cada doctorando fue clasificado según el sexo, la obtención del título de doctor y la fecha de finalización de la tesis. Una vez obtenidos los datos se realizaron los análisis del rendimiento global y la distribución de los doctorandos en las diferentes áreas de conocimiento según el sexo de los doctorandos.

\section{RESULTADOS}

Se realizó el análisis del rendimiento diferencial por sexo. En el grupo de las mujeneres el 41,7\% logró el título de doctor mientras que en el grupo de los hombres estos fueron el $40 \%$. Se efectuó la prueba chi-cuadrado y resultó que los dos grupos no difieren de manera estadísticamente significativa $\left(\mathrm{X}_{(1 ; \mathrm{n}=3463)}^{2}=1,014 ;(p=0,314)\right.$.

Seguidamente se repitieron los análisis de forma separada para cada una de las áreas de conocimiento establecidas por el Consejo de Coordinación Universitaria. Para ello únicamente se utilizaron los becarios FPU, ya que en los datos de los FPI no se recoge información sobre el área de conocimiento de los mismos. Así pues, se analizó la frecuencia de concesiones por área y sexo (Tabla 2). En ella se observa que en humanidades, ciencias experimentales y ciencias sociales y jurídicas no hay apenas diferencias, mientras, en las enseñanzas técnicas hay un $22 \%$ más de hombres que mu- 


\begin{tabular}{|c|c|c|c|c|c|}
\hline \multicolumn{6}{|c|}{ Becarios Predoctorales (2002-2003) } \\
\hline & Doctorados & No Doctorados & Total & ${ }_{-2}^{2}(\mathrm{~g} \cdot \mathrm{l} ; \mathrm{n})$ & $\mathrm{p}$ \\
\hline Hombres & $696(40,0 \%)$ & $1.043(60,0 \%)$ & $1.739(100 \%)$ & $1,014(1 ; 3463)$ & 0,314 \\
\hline Mujeres & $719(41,7 \%)$ & $1.005(58,3 \%)$ & $1.724(100 \%)$ & & \\
\hline Total & $1.415(40,9 \%)$ & $2.048(59,1 \%)$ & $3.463(100 \%)$ & & \\
\hline
\end{tabular}

Tabla 1. Frecuencia (porcentaje) doctorados entre el año 2002 y 2009 según sexo (de las convocatorias de becas FPU y FPI de los años 2002 y 2003).

jeres. Sucede lo contrario en las ciencias de la salud, donde hay un 38\% más de mujeres. Para comprobar estadísticamente si estas diferencias son significativas se recurrió a la chi-cuadrado. Esta prueba confirmó que hay diferencias estadísticamente significativas $\left(\chi_{(4 ; n=1946)}^{2}=35,290 ; p=0,000\right)$ en las concesiones de las becas FPU en función del sexo y del área de conocimiento.

\begin{tabular}{|l|c|c|c|}
\hline \multicolumn{1}{|c|}{ Área } & Hombre (\%) & Mujer (\%) & Total \\
\hline Humanidades & $165(46,3)$ & $191(53,7)$ & 365 \\
\hline Ciencias experimentales & $442(49,8)$ & $446(50,2)$ & 888 \\
\hline Ciencias sociales y jurídicas & $129(48,1)$ & $139(51,9)$ & 268 \\
\hline Enseñanzas técnicas & $189(61)$ & $121(39)$ & 310 \\
\hline Ciencias de la salud & $39(31)$ & $87(69)$ & 126 \\
\hline
\end{tabular}

Tabla 2. Frecuencia (porcentaje) de becas FPU por área y sexo (convocatoria 2002 y 2003)

Una vez vistas las diferencias en las concesiones se pasó al análisis del rendimiento diferencial por área y sexo (Tabla 3). Para realizar las comparaciones por sexo se calcularon cinco chi-cuadrado (una por cada área de conocimiento). En los resultados se observa que no hay diferencia en el rendimiento de hombres y mujeres en cada una de las áreas de conocimiento. 
M. P. BERMÚDEZ, A. GUILLÉN-RIOUELME, A. GÓMEZ-GARCÍA, R. QUEVEDO-BLASCO, J. C. SIERRA, G. BUELA-CASAL ANÁLISIS DEL RENDIMIENTO EN EL DOCTORADO EN FUNCIÓN DEL SEXO

\begin{tabular}{|c|c|c|c|c|c|}
\hline Área & Sexo & $\begin{array}{l}\text { Doctor } \\
\text { Sí }(\%)\end{array}$ & No $(\%)$ & $\chi^{2 *}$ & $\mathrm{p}^{* *}$ \\
\hline Humanidades & $\begin{array}{l}\text { Hombre } \\
\text { Mujer } \\
\text { Total }\end{array}$ & $\begin{array}{l}35(21,2) \\
48(25,1) \\
83(23,3)\end{array}$ & $\begin{array}{l}130(78,8) \\
143(74,9) \\
273(76,7)\end{array}$ & $0,760(1)$ & 0,383 \\
\hline $\begin{array}{c}\text { Ciencias } \\
\text { experimentales }\end{array}$ & $\begin{array}{l}\text { Hombre } \\
\text { Mujer } \\
\text { Total } \\
\end{array}$ & $\begin{array}{c}192(43,4) \\
183(41) \\
375(42,2) \\
\end{array}$ & $\begin{array}{c}250(56,6) \\
263(59) \\
513(57,8) \\
\end{array}$ & 0,527 (1) & 0,468 \\
\hline $\begin{array}{l}\text { Ciencias } \\
\text { sociales y } \\
\text { jurídicas }\end{array}$ & $\begin{array}{l}\text { Hombre } \\
\text { Mujer } \\
\text { Total }\end{array}$ & $\begin{array}{l}34(26,4) \\
36(25,9) \\
70(26,1)\end{array}$ & $\begin{array}{c}95(73,6) \\
103(74,1) \\
198(73,9)\end{array}$ & 0,007 (1) & 0,932 \\
\hline $\begin{array}{c}\text { Enseñanzas } \\
\text { técnicas }\end{array}$ & $\begin{array}{l}\text { Hombre } \\
\text { Mujer } \\
\text { Total }\end{array}$ & $\begin{array}{c}87(46) \\
65(53,7) \\
152(49)\end{array}$ & $\begin{array}{c}102(54) \\
56(46,3) \\
158(51)\end{array}$ & $1,744(1)$ & 0,187 \\
\hline $\begin{array}{l}\text { Ciencias de } \\
\text { la salud }\end{array}$ & $\begin{array}{l}\text { Hombre } \\
\text { Mujer } \\
\text { Total }\end{array}$ & $\begin{array}{c}17(43,6) \\
41(47,1) \\
58(46)\end{array}$ & $\begin{array}{c}22(56,4) \\
46(52,9) \\
68(54)\end{array}$ & $0,136(1)$ & 0,713 \\
\hline
\end{tabular}

*Grados de libertad = 1 para todas las comparaciones. $* * \mathrm{p}=$ probabilidad asociada a la chi-cuadrado.

Tabla 3. Comparación de doctores por área y sexo entre los becarios FPU de las convocatorias 2002 y 2003

En los resultados se observa que no hay diferencias significativas en el rendimiento de hombres y mujeres en cada una de las áreas de conocimiento (Figura 2).

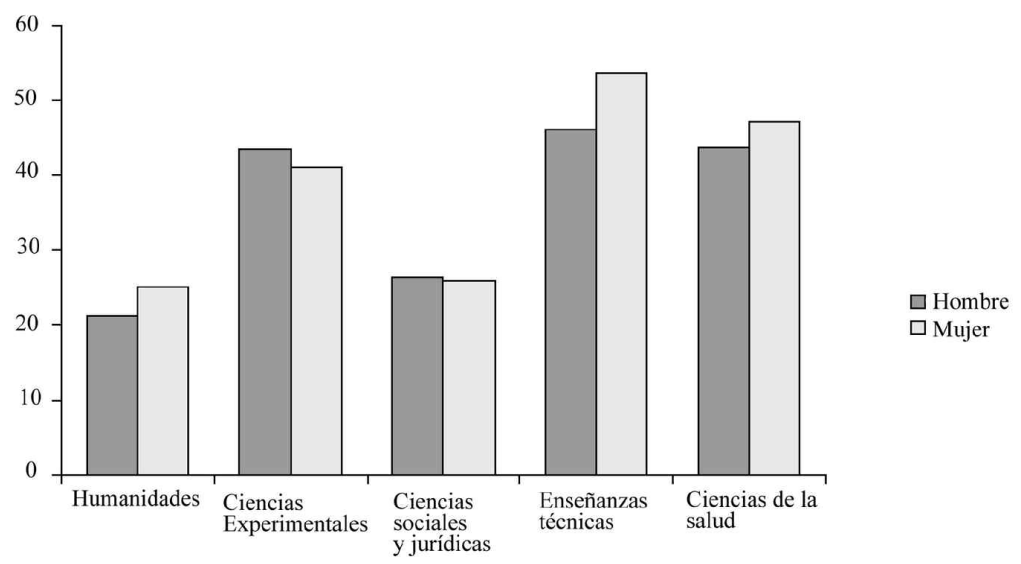

Figura 2. Comparación del porcentaje de doctores becados FPU del 2002 y 2003 por áreas de conocimiento según el sexo en le periodo 2002-2009. 


\section{DISCUSIÓN}

Como se ha expuesto en los resultados, no se evidencia ninguna diferencia significativa en el número de tesis doctorales realizadas por hombres y mujeres, demostrando la misma tasa de éxito. Otro resultado interesante hallado en este trabajo es que hombres y mujeres se distribuyen de manera homogénea en las áreas de humanidades, ciencias experimentales y ciencias sociales y jurídicas pero no se encuentra la misma distribución en las áreas de las enseñanzas técnicas y de ciencias de la salud. Un estudio previo obtuvo resultados parecidos ya que halló equidad por sexo en las tesis doctorales presentadas entre el 1990 y el 2004 (Villarroya, Barrios, Borrego, Frías, 2008). Por el contrario, en otros trabajos se observó que, a pesar de que el número de las mujeres matriculadas en el doctorado supera al de los hombres, el porcentaje de las que consiguen el título de doctora es menor (Pérez et al., 2003). Además, según el INE las mujeres matriculadas en el doctorado constituyen el $52 \%$ del total, mientras que la tasa de doctoras es un poco inferior al de los hombres (49\%) (INE, 2009). No obstante, en este estudio no se igualaron las condiciones económicas.

Algunos autores se preguntan si la desigualdad de representación de los dos sexos en la ciencia sea debida a una diferencia real en la producción científica. En un estudio de Bermúdez, Castro y Buela-Casal (2007), donde se analiza la producción científica de hombres y mujeres pertenecientes a las universidades españolas incluidas en el Academic Ranking of World Universities (Institute of Higher Education, Shanghai Jiao Tong University, 2008) se evidencia que las mujeres tienen un número de tramos de investigación superior al de los hombres. Las mujeres resultan ser también más exigentes respecto a los criterios para evaluar la carrera científica y valoran más un modelo de selección de tipo meritocrático para acceder a los cuerpos docentes universitarios respecto a los hombres (Sierra, Buela-Casal, Bermúdez y Santos-Iglesias, 2009a). En otros resultados obtenidos por Bordons, Morillo, Fernández y Gómez (2003) donde no se encuentra ninguna diferencia significativa entre hombres y mujeres en la producción científica en las áreas de ciencias naturales y química de los investigadores del CSIC. Además, los intentos por explicar la presencia desigual de la mujer en ciertos ámbitos científicos con teorías referidas a la diversidad sexual en habilidades cognitivas no han logrado su objetivo, ya que se ha demostrado que no hay alguna diferencias entre sexos en cuanto a las habilidades matemáticas (Hyde, Lindberg, Linn, Ellis, Williams, 2008). Otros autores intentan comprender esta desigualdad a partir de los procesos de socialización. El estereotipo relativo a que las niñas y las mujeres tienen mayores dificultades que los hombres con la aritmética persiste entre los padres e influye muchísimo tanto en las actitudes (Jacobs y Eccles, 1992), como en las futuras elecciones del tipo de estudios de los hijos (Bleeker y Jacobs, 2004). El hecho de que no haya modelos femeninos de referencia en 
el mundo de la ciencia podría ser otro motivo de desmotivación por parte de las chicas que podrían emprender carreras científico-técnicas (Blickenstaff, 2005). Otros autores explican la desigualdad de representación de los dos sexos en la ciencia con el hecho de que el conflicto entre vida laboral y familiar afecta más la vida profesional de las científicas y académicas que la de los hombres (Wolfinger, Mason y Goulden, 2009).

A partir de los resultados del presente estudio, parece que cuando las doctorandas tienen dedicación plena a su trabajo de doctorado, gracias a ser beneficiarias de una beca predoctoral, no existen diferencias en la tasa de éxito por sexo. Sin embargo, aquellas mujeres que no perciben ayudas específicas para conseguir el doctorado tienen más dificultades que los hombres para compatibilizar sus estudios, sus profesiones o sus responsabilidades familiares, por lo que su rendimiento es menor. 


\section{NOTAS}

1. El estudio ha sido financiado parcialmente por la Dirección General de Universidades. Referencia: EA2008-0208. 


\section{REFERENCIAS BIBLIOGRÁFICAS}

Agudelo, D., Bretón-López, J., Ortiz-Recio, G., Poveda-Vera, J., Teva, I., ValorSegura, I. y Vico, C. (2003). Análisis de la productividad científica de la Psicología española a través de las tesis doctorales. Psicothema, 15, 595-609.

Asamblea General de las Naciones Unidas. (1945). Carta de las Naciones Unidas. Disponible en: http://www.un.org/ es/documents/charter/intro.shtml. [Consulta 2009, 20 de abril].

Asamblea General de las Naciones Unidas. (1948). Declaración Universal de los Derechos Humanos. Disponible en: http://www.un.org/es/documents/udhr/. [Consulta 2009, 20 de abril].

Bermúdez, M.P., Castro, A. y Buela-Casal, G. (2007). Análisis de la productividad científica por género en las mejores universidades españolas, en $\mathrm{M}$. P. Bermúdez y A. Castro (Comps.), Evaluación de la calidad de la educación superior y la investigación científica (IV Foro) Granada: Fundación Empresa-Universidad de Granada, 81.

Bermúdez, M. P., Castro, A., Sierra, J. C. y Buela-Casal, G. (2009). Análisis descriptivo transnacional de los estudios de doctorado en el EEES. Revista Psicodidáctica, 14, 193-210.

Bleeker, M. M. y Jacobs, J. E. (2004). Achievement in math and science: do mothers' beliefs matter 12 years later? Journal of Educational Psychology, 96, 97-109.

Blickenstaff, J. C. (2005). Women and science careers: leaky a pipeline or gender filter? Gender and Education, 17, 369-386.

Bordons, M., Morillo, F., Fernández M. T. y Gómez, I. (2003). One step further in the production of bibliometric indicators at the micro level: differences by gender and professional category of scientists. Scientometrics, 57, 159-173.

Bretz, B. (2010). Análisis de las fortalezas institucionales para la contratación de investigadores. Aula Abierta, 38, 6574 .

Buela-Casal, G. (2005). Recomendaciones para superar un programa de doctorado, en G. Buela-Casal (Dir.), Manual práctico para hacer un doctorado. Madrid: Eos, 153-167.

Buela-Casal, G. (2010). Índices de impacto de las revistas científicas e indicadores para medir el rendimiento de los investigadores. Revista de Psicodidáctica, 15, 3-19.

BuelaCasal, G. y Castro, A. (2008a). Análisis de la evolución de los Programas de Doctorado con Mención de Calidad en las universidades españolas y propuestas de mejora. Revista de Investigación en Educación, 5, 496.

Buela-Casal, G. y Castro, A. (2008b). Criterios y estándares para la obtención de la Mención de Calidad en Programas de Doctorado: Evolución a través de las convocatorias. International Journal of Psychology and Psychological Therapy, 8, 127-136

Buela-Casal, G. y Sierra, J. C. (2007). Criterios, indicadores y estándares para la acreditación de profesores titulares y catedráticos de Universidad. Psicothema, 19, 537-551.

Castro, A. y Buela-Casal, G. (2008). La movilidad de profesores y estudiantes en programas de postgrado: ranking de las universidades españolas. Revista de Investigación en Educación, 5, 61-74. 
Castro, A., Guillen-Riquelme, A., Quevedo-Blasco, R. Ramiro, M. T., Bermúdez, M. P. y Buela-Casal, G. (2010). Las Escuelas Doctorales: evolución histórica, características y aspectos relevantes para su consolidación en España. Aula Abierta, 38, 17-28.

Consejo Económico y Social de las Naciones Unidas. (1979). Convención sobre la eliminación de todas las formas de discriminación contra las mujeres. Disponible en: http://www.un.org/womenwatch/daw/cedaw/text/sconvention.htm. [Consulta 2009, 18 de junio].

Consejo Superior de Investigación Científica (2002). Proposición de la constitución de la Comisión Mujeres y Ciencia del CSIC. Disponible en: http://www.csic.es/mujerCiencia.do. [Consulta 2009, 20 de junio].

Consejo Superior de Investigación Científica (2009). Informe mujeres investigadoras 2009. Mujeres y Ciencia. Disponible en:

http://www.csic.es/documentos/mujerCiencia/informe_mujeres_2009.pdf. [Consulta 2009, 5 de abril].

De Pablo, F. (2004). En la ciencia todavía no existe el tanto monta-monta tanto. Torre de los Lujanes: Boletín de la Real Sociedad Económica Matritense de Amigos del País, 53, 81-90.

Fundación Española para la Ciencia y la Tecnología. (2005). Mujeres y ciencia. La situación de las mujeres investigadoras en el sistema español de ciencia y tecnología. Disponible en: http://www.fecyt.es/fecyt/seleccionarMenu2.do?strRutaNivel2=;Publicaciones;EstudiosInformes\&strRutaNivel1 $=$;Publicaciones\&tc $=$ publicaciones . [Consulta 2009, 21 de marzo].

González-Ramos, A. M., González de la Fe, T., Fernández-Esquinas, M., PeñaVázquez, R. Bonnet-Escuela, M. y Van
Oostrom, M. (2006). Política de recursos humanos y carrera investigadora. Memoria del proyecto EA2006-0099, BOE 11 de abril 2006. Disponible en: http://www.centrorecursos.com/mec/ay udas/CasaVer.asp?P=29 221 . [Consulta 2008, 15 diciembre].

Guillen-Riquelme, A., Guglielmi, O., Ramiro, M. T., Castro, A. y Buela-Casal, G. (2010). Rendimiento en el doctorado de los becarios FPU y FPI en el Consejo Superior de Investigaciones Científicas y en las universidades públicas españolas. Aula Abierta, 38, 7582.

Handschin, C. H. (1911). Academic and industrial efficiency. Science, 9, 892893.

Hyde, J. S., Lindberg, S. M., Linn, M. C., Ellis, A. B. y Williams, C. C. (2008). Gender similarities characterize math performance. Science, 25, 494-495.

Institute of Higher Education, Shanghai Jiao Tong University. (2008). Academic Ranking of World Universities. Disponible en:

http://ed.sjtu.edu.cn/rank/2008/2008Ma in.htm. [Consulta 2009, 20 de enero].

Instituto Nacional de Estadística. (2009). Estadística de la Enseñanza Universitaria en España. Curso 2007-2008. Disponible en:

http://www.ine.es/jaxi/menu.do?type $=\mathrm{p}$ caxis \& path $=/$ t $13 /$ p $405 /$ a 2007 2008\&file $=$ pcaxis. [Consulta 2009, 20 de marzo].

Jacobs, J. E. y Eccles, J. S. (1992). The impact of mothers' gender-role stereotypic beliefs on mothers' and children's ability perceptions. Journal of Personality and Social Psychology, 63, 932-944.

Jefatura del Estado. Ley Orgánica 3/2007, de 22 de marzo, para la igualdad efecti- 
va de mujeres y hombres. BOE núm. 71 de 23 de marzo de 2007. Disponible en: http://www.boe.es/aeboe/consultas/bases_datos/doc.php?coleccion=ibe rlex\&id=2007/06115. [Consulta 2009, 20 de junio].

Ministerio de Educación y Ciencia (2007). Académicas en cifras 2007. Disponible en: http://ciencia.micinn.fecyt.es/ciencia/umyc/files/2007-academicas-en-cifras.pdf. [Consulta 2009, 23 de abril].

Ministerio de la Presidencia. Orden APU/256/2005, de 7 de marzo, por la que se dispone la publicación del Acuerdo de Consejo de Ministros de 4 de marzo de 2005, por la que se aprueba el plan para la igualdad de género en la Administración General del Estado. BOE núm. 57 de 8 de marzo de 2005. Disponible en:

http://www.boe.es/boe/dias/2005/03/08/ pdfs/A08111-08114.pdf. [Consulta 2009, 23 de abril].

Montero, I. y León, O. G. (2007). A guide for naming research studies in Psychology. International Journal of Clinical and Health Psychology, 7, 847-862.

Moyano, M., Delgado, C. J. y BuelaCasal, G. (2006). Análisis de la productividad científica de la Psiquiatría española a través de las tesis doctorales en la base de datos TESEO (19932002). International Journal of Psychology and Psychological Therapy, 6, 111-120.

Musi-Lechuga, B., Olivas-Ávila, J. A. y Buela-Casal, G. (2009). Producción científica de los programas de doctorado en Psicología Clínica y de la Salud en España. International Journal of Clinical and Health Psychology, 9, 161-173.

Olivas-Ávila, J. A. y Musi-Lechuga, B. (2010). Producción en tesis doctorales de los profesores funcionarios de psicología en España más productivos en la Web of Science. Psicothema, 22, 917-923.

Organización para la Cooperación y el Desarrollo Económico. (2003). Informe PISA 2003. Disponible en: http://www.oecd.org/dataoecd/59/1/397 32493.pdf. [Consulta 2009, 24 de mayo].

Osborn, M. (2001). Promover la excelencia mediante la integración de la igualdad entre géneros. Luxemburgo: Oficina de Publicaciones Oficiales de las Comunidades Europeas.

Pérez, E. (1996). De la biología imaginaria a la sociología real: obstáculos para el acceso de las mujeres a la ciencia, en M. A. García de León, M. García de Cortázar y F. Ortega (Eds.), Sociología de las mujeres españolas. Madrid: Editorial Complutense, 219-242.

Pérez, E., González, M., Miranda, M. J., Ortega, E. y Sanz, V. (2006). La cuestión de género en la investigación española, en J. Sebastián y E. Muñoz (Eds.), Radiografía de la investigación pública en España. Madrid: Biblioteca Nueva, 122-147.

Pérez, E., González, M., Santesmases, M. J., Martín, A., de Villota, P. y Guill, A. (2003). La situación de las mujeres en el sistema educativo de ciencia y tecnología en España y su contexto internacional. Disponible en:

http://www.csic.es/documentos/mujerCiencia/2003_Mujeres_en_CyT_en_Espana.pdf. [Consulta 2009, 19 de marzo].

Quevedo-Blasco, R., Del Río, L., Bermúdez, M. P. y Buela-Casal, G. (2010). Relación entre los programas de doctorado con Mención de Calidad y la productividad de tesis doctorales. Revista Digital Universitaria, 11, 1-14. 
Ramos-Álvarez, M. M., Moreno-Fernández, M. M., Valdés-Conroy, B. y Catena, A. (2008). Criteria of the peer-review process for publication of experimental and quasi-experimental research in Psychology: a guide for creating research papers. International Journal of Clinical and Health Psychology, 8, 751-764.

Sierra, J. C., Buela-Casal, G., Bermúdez, M. P. y Santos-Iglesias, P. (2009a). Diferencias por sexo en los criterios y estándares de productividad científica y docente en profesores funcionarios en España. Psicothema, 21, 124-132.

Sierra, J. C., Buela-Casal, G., Bermúdez, M. P. y Santos-Iglesias. P. (2009b). Im- portancia de los criterios e indicadores de evaluación y acreditación del profesorado funcionario universitario en los distintos campos de conocimiento de la UNESCO. Revista de Universidad y Sociedad del Conocimiento (RUSC), 6, 49-59.

Villarroya, A., Barrios, M., Borrego, A. y Frías, A. (2008). PhD theses in Spain: A gender study covering the years 19902004. Scientometrics, 77, 469-483.

Wolfinger, N. H., Mason, M. A. y Goulden, M. (2009). Stay in the game: gender, family formation and alternative trajectories in the academic life course. Social Force, 87, 1591-1621. 


\section{PALABRAS CLAVE}

Educación superior, estudios de doctorado, mujeres, ciencia.

\section{KEYWORDS}

Higher Education, PhD studies, Women, Science.

\section{PERFIL ACADÉMICO Y PROFESIONAL DE LOS AUTORES}

M. Paz Bermúdez, Licenciada y Doctora en Psicología, en la actualidad Profesora Titular del Departamento de Psicología Evolutiva y de la Educación de la Universidad de Granada. Su principal línea de investigación se desarrolla en el campo de la prevención del VIH con población adolescente. Es investigadora principal de varios proyectos de investigación subvencionados, entre otros organismos, por el Ministerio de Asuntos Exteriores, el Ministerio de Ciencia e Innovación y la Dirección General de Universidades.

Alejandro Guillén-Riquelme, Personal Docente Investigador del Departamento de Personalidad, Evaluación y Tratamientos Psicológicos en la Facultad de Psicología de la Universidad de Granada. Sus investigaciones se centran principalmente en la evaluación de la ansiedad y el estudio de variables que influyen en la calidad de la educación superior y la investigación.

Almudena Gómez-García, Personal Docente Investigador del Departamento de Personalidad, Evaluación y Tratamientos Psicológicos, en la Facultad de Psicología de la Universidad de Granada. Desarrolla sus investigaciones en el ámbito de la evaluación de programas y la evaluación de la educación superior y la investigación.

Raúl Quevedo-Blasco, Personal Docente Investigador del Departamento de Personalidad, Evaluación y Tratamientos Psicológicos, en la Facultad de Psicología de la Universidad de Granada. Sus ámbitos de estudio son, entre otros, la apnea del sueño y la evaluación de la educación superior y la investigación.

Juan Carlos Sierra, Doctor en Psicología y Profesor Titular de Evaluación Psicológica en la Universidad de Granada. Sus líneas de investigación se centran en el área de la sexualidad, el sueño y la evaluación de la ciencia. Ha editado o coeditado siete monografías sobre estas temáticas y publicado un centenar de artículos científicos. Es el actual editor del International Journal of Clinical and Health Psychology. 
Gualberto Buela-Casal, Catedrático de Psicología de la Personalidad, Evaluación y Tratamientos Psicológicos en la Facultad de Psicología de la Universidad de Granada. Entre sus líneas de investigación destaca la apnea del sueño y su relación con otras variables, el VIH/Sida, la impulsividad/reflexividad y la evaluación de la educación superior y la investigación, en lo que respecta a evaluación de profesorado, másteres con mención de calidad y escuelas doctorales, entre otros.

Dirección de los autores: Facultad de Psicología. Universidad de Granada. e-mail: maripaz@ugr.es

Fecha de Recepción del artículo: 14. Diciembre. 2010

Fecha de Aceptacion del artículo: 16. Diciembre. 2010 
